\title{
Emergent horizon, Hawking radiation and chaos in the collapsed polymer model of a black hole
}

\author{
Ram Brustein ${ }^{(1)}$, A.J.M. Medved ${ }^{(2,3)}$
}

\begin{abstract}
(1) Department of Physics, Ben-Gurion University, Beer-Sheva 84105, Israel
(2) Department of Physics \& Electronics, Rhodes University, Grahamstown 6140, South Africa

(3) National Institute for Theoretical Physics (NITheP), Western Cape 7602, South Africa

ramyb@bgu.ac.il, j.medved@ru.ac.za
\end{abstract}

\begin{abstract}
We have proposed that the interior of a macroscopic Schwarzschild black hole (BH) consists of highly excited, long, closed, interacting strings and, as such, can be modeled as a collapsed polymer. It was previously shown that the scaling relations of the collapsed-polymer model agree with those of the BH. The current paper further substantiates this proposal with an investigation into some of its dynamical consequences. In particular, we show that the model predicts, without relying on gravitational effects, an emergent horizon. We further show that the horizon fluctuates quantum mechanically as it should and that the strength of the fluctuations is inversely proportional to the $\mathrm{BH}$ entropy. It is then demonstrated that the emission of Hawking radiation is realized microscopically by the quantum-induced escape of small pieces of string, with the rate of escape and the energy per emitted piece both parametrically matching the Hawking temperature. We also show, using standard methods from statistical mechanics and chaos theory, how our model accounts for some other known properties of BHs. These include the accepted results for the scrambling time and the viscosity-to-entropy ratio, which in our model apply not only at the horizon but throughout the $\mathrm{BH}$ interior.
\end{abstract}




\section{Introduction}

The traditional narrative, based on classical general relativity, is that the interior of a Schwarzschild black hole $(\mathrm{BH})$ is a region of mostly empty space surrounding a highly dense, very small and classically singular core. It has recently been suggested that this picture is not only flawed but spectacularly incorrect. This current narrative can be simply summarized: There is no interior to even consider. Spacetime rather ends at the BH horizon either as a matter of principle, as in the fuzzball model of BHs [1, 2, 3, 4] (also [5]), or in practice, as a "firewall" of high-energy particles surrounding the horizon [6] (also [7, 8, 9]). We, however, have been advocating a rather different point of view: The interior exists and is not empty; it is rather filled with a hot bath of long, closed, interacting strings [10]. Such an interior cannot be described in terms of a semiclassical spacetime metric, and we have proposed that the appropriate description of this state is in terms of a collapsed polymer.

The basis for our reasoning is that the interior of a $\mathrm{BH}$ must be in a highly quantum state when it is described in terms of the spacetime fields at infinity [11]. Accepting that BHs evolve in a unitary manner and that they can be in a (nearly) pure state, it follows that the interior state must be the purifier of the emitted Hawking radiation. Therefore, the non-vanishing eigenvalues of the density matrix of the emitted radiation and those of the interior state are equal. The emitted radiation is in a highly quantum state because the emission rate of $\mathrm{BHs}$ is equal to the inverse of the wavelength of the emitted radiation. This is distinct from standard black bodies, such as the Sun, for which the emission rates are much larger than the inverse of the radiation wavelengths. Therefore, the emitted radiation from $\mathrm{BHs}$ is charac- 
terized by its small occupation numbers in the basis of the asymptotic fields. It follows that the state of the interior must, similarly, posses small occupation numbers. Further, if a mean-field description of this state does exist, it must be in terms of a strongly correlated system or, equivalently, an entropically dominated one to account for the BH's unorthodox thermodynamics; in particular, its non-extensive entropy and energy.

Once it is accepted that the $\mathrm{BH}$ interior does have such a highly quantum description, one is almost inevitably led to a high-temperature theory of fundamental strings. As has been frequently discussed in the literature (e.g., [12, 13, 14, 15, 16, 17]), the partition function for such a theory has an exponentially large number of closely packed states and its canonical ensemble is subject to large fluctuations that diverge at the Hagedorn temperature. In other words, this is a theory of highly quantum matter. Moreover, the equation of state for high-temperature string theory is famously $p=\rho$, which is equivalent to $s=\sqrt{\rho}$ in suitable units. Here $p, \rho$ and $s$ are respectively pressure, energy density and entropy density. Thus, $s$ is as large as it can be in comparison to $\rho$, implying entropic dominance.

The $\mathrm{BH}$ has the same equation of state, $s=\sqrt{\rho}$, if the interior matter is distributed so as to saturate the Bekenstein-Hawking entropy-area law [18, 19] for any interior, closed surface [20]. The same equation of state also signals the saturation of the causal entropy bound [21] which implies, in turn, the breakdown of semiclassical physics [22]. In this way, one can see how the notions of highly quantum and entropically dominant are indeed synonymous.

What we have proposed is that the $\mathrm{BH}$ interior should be modeled as a 
Schwarzschild-sized, bound and metastable (but classically stable) state of interacting, long, closed strings at a temperature approaching the Hagedorn temperature from above. Exactly at the Hagedorn temperature, the free energy identically vanishes and consequently the entropy density and the energy density are equal, $s=\rho$. As just pointed out, we are considering a high-temperature phase approaching the Hagedorn temperature from above. For this phase, Atick and Witten [14] showed that the free-energy density scales as $F / V \sim T^{2}$, which implies $\rho=E / V \sim T^{2}$ and $s=S / V \sim T$. Hence, up to an order-unity numerical factor, $s=\sqrt{\rho}$ is indeed the relation between the entropy density and the energy density.

As discussed at length in [23, 24], summarized in [10] and reviewed in the next section, the resulting picture is one in which the bound state is dominated by $\ln N$ long strings, where $N$ is the total length of the strings in string units (or, equivalently, the number of "string bits"). This picture differs somewhat from the commonly held view that a single long string is entropically favored. The latter scenario applies only when the strings are placed in a very large volume. The difference then comes about from finitevolume effects modifying the corresponding equilibrium state.

Our proposal is different than the traditional BH-string correspondence [25, 26, 27], for which the duality between $\mathrm{BH}$ and string thermodynamics depends on having a $\mathrm{BH}$ whose Schwarzschild radius $R_{S}$ matches the fundamental string length $l_{s}$. But this requirement cannot possibly apply to macroscopic BHs. The main difference between our proposal and the BH-string correspondence is that, in our setup, there are two distinct scales of energy. One scale is the string-tension energy, which is given by 
$E_{t e n}=N / l_{s}$ and is the microscopic energy that would be attributed to a single free string of total length $\mathrm{Nl}_{s}$. This energy scale is related via the first law to the Hagedorn temperature $T_{H a g}=1 / l_{s}$ and the string entropy $N$. 1 The other scale is the bound-state energy $E_{\text {bound }}$ which, together with the tension energy, accounts for the interaction energy $E_{\text {int }}$ of the strings, $E_{\text {bound }}=E_{\text {ten }}-E_{\text {int }}$. This is the energy that an external observer measures, having no direct access to the interior, and it is the energy associated with the Hawking temperature $T_{\text {Haw }}=E_{\text {bound }} / N$. So that, if $l_{P}$ is the Planck length and $M_{B H}=\left(1 / l_{P}\right)\left(R_{S} / l_{P}\right)^{d-2}$ is the mass of the $\mathrm{BH}$, our proposal requires that $M_{B H}=E_{\text {bound }}<E_{\text {ten }}$, rather than a correspondence point where $M_{B H}=E_{t e n}$ as in [25, 26, 27].

We would now like to go beyond the thermodynamic scaling relations of our previous work and find out whether other known features of BHs are shared by the collapsed-polymer model. This is the purpose of the current paper.

The most prominent feature of a $\mathrm{BH}$ is that it has a horizon. By "horizon", we mean a generic term describing a classically causal barrier to the external world. In semiclassical gravity, the horizon is described from the exterior of the $\mathrm{BH}$ in geometric terms, and one can make the distinction among the various geometric descriptions such as an event horizon, a particle horizon or an apparent horizon. In our model, a geometric description of the interior is lacking, but one can still adopt the equivalent representation of gravity as a dynamical effect in flat space. Flat-space coordinates, $t$,

\footnotetext{
${ }^{1}$ We mostly leave out order-unity numerical factors and fundamental constants, often set $l_{s}=1$ and assume $d+1$ dimensions with $d \geq 3$.
} 
$r$, etc., would then represent fiducial coordinates; essentially, labels for the constituent objects (strings). From this point of view, gravity is emergent; it is an effective macroscopic description of the microscopic forces between constituents. The geometric description (if it exists) can replace the macroscopic gravitational force. When gravity is semiclassical, both geometric and dynamical descriptions co-exist.

Lacking a geometrical description of the spacetime, we have chosen the dynamical representation and applied it throughout the paper. What we would then like to understand is if (and how) a horizon emerges from the perspective of the polymer. We find that the casual barrier can be described in the fiducial coordinates of flat space as (approximately) a surface of constant $r=R_{S}$ from which pieces of string cannot escape classically.

Further, as was argued by Bekenstein [18] and later proved by Hawking, matter can still break through this causal barrier but can only do so quantum mechanically. This rasies the question of how, for our model, does the quantum-mechanical emission of Hawking radiation come about. A crucial consistency check for the polymer is that the emission of Hawking particles should occur at both a rate and energy release that matches $T_{\text {Haw }}$. We would also like to know if the emergent horizon fluctuates quantum mechanically and, if so, compare the strength of these fluctuations to that of previous works [28, 29, 30].

Another distinctive feature of $\mathrm{BHs}$ is that they are maximally fast scramblers of information [31, 32, 33]. This leads to another important litmus test for our model: It must reproduce the minimally allowed scrambling time. This is basically the same as realizing the maximum possible value of the 
Lyapunov exponent, which also makes BHs the most chaotic of all systems [34. Closely related to the idea of a BH being an ideal scrambler and maximally chaotic is that its viscosity-to-entropy ratio is minimized [35, 36, 37, thus providing another essential check.

After a review of our proposed model, the remainder of the paper puts it to the test by investigating the questions posed above. We find that it passes all of them with "flying colors", providing a direct and simple physical explanation in all cases.

\section{Review of the polymer model}

Much of the content of this section has been already covered in [10]. We include it here for completeness.

\subsection{Highly excited strings in a bounded region}

We wish to describe a bound state of long closed strings. The idea is that the closed strings occupy a bounded region in space whose size is determined dynamically. A more figurative description of the proposed bound state is that of a "quantum star" made of fundamental strings in the Hagedorn phase or simply a "string ball".

We start our discussion by following the descriptions of Salomonson and Skagerstam (SS) [23], and Lowe and Thorlacius (LT) [24] of highly excited strings in a bounded region. Their description relies on two properties of interacting, long strings: (1) That the total length is conserved due to the conservation of energy and (2) that the probability for interaction is pro- 
portional to the length of the string. In the particular setting of the SS-LT model — where interactions are described by the splitting and joining of strings — only the three-string interactions are important, while the fourstring and higher-order interactions can be neglected. The SS-LT model was later validated by detailed investigations using the "thermal-scalar" formalism [17], the world-sheet description of (open) bosonic strings [38] and of closed-strings [39].

The LT description is particularly useful for motivating our proposal for the $\mathrm{BH}$ interior. These authors considered a high-temperature collection of interacting, closed strings in a finite volume such that the total length of string is much longer than the spatial dimensions of the enclosing volume. They consider the volume of the confining region or "box" to be fixed; for example, strings moving on a toroidal space of a fixed size. We regard the finite volume of the bound state as being due to the (attractive) interactions of the strings. So that, in our case, the interaction energy is parametrically the same as the string tension. As discussed later, it is also true for our case that the three-string interactions are important, and the four-string and higher-order interactions can be neglected.

Let us briefly sketch the pertinent results of LT, who considered the Boltzmann equation for long, closed strings when only three-string interactions are relevant. In $l_{s}=1$ units, this is [24]

$$
\begin{aligned}
\frac{d n(\ell)}{d t}=k \frac{g^{2}}{V}[ & -\frac{1}{2} \ell^{2} n(\ell)-\int_{0}^{\infty} d \ell^{\prime} \ell^{\prime} n\left(\ell^{\prime}\right) \ell n(\ell) \\
& \left.+\frac{1}{2} \int_{0}^{\ell} d \ell^{\prime} \ell^{\prime}\left(\ell-\ell^{\prime}\right) n\left(\ell^{\prime}\right) n\left(\ell-\ell^{\prime}\right)+\int_{\ell}^{\infty} d \ell^{\prime} \ell^{\prime} n\left(\ell^{\prime}\right)\right]
\end{aligned}
$$

where $n(\ell)$ is the average number of strings of length $\ell, k$ is a numerical 
factor and each of the four terms on the right represents a different type of three-string interaction. See Fig. 1, The first term represents the possibility that a string of length $\ell$ splits into two shorter ones, while the second term accounts for two strings of lengths $\ell$ and $\ell^{\prime}$ joining together to form one of length $\ell+\ell^{\prime}$. The third term results from two shorter strings joining to make a longer string of length $\ell$ and, finally, the last term accounts for a longer loop splitting into two shorter ones, one of which is of length $\ell$. Higher-order interactions are suppressed by the smallness of the string coupling and/or the largeness of the volume.

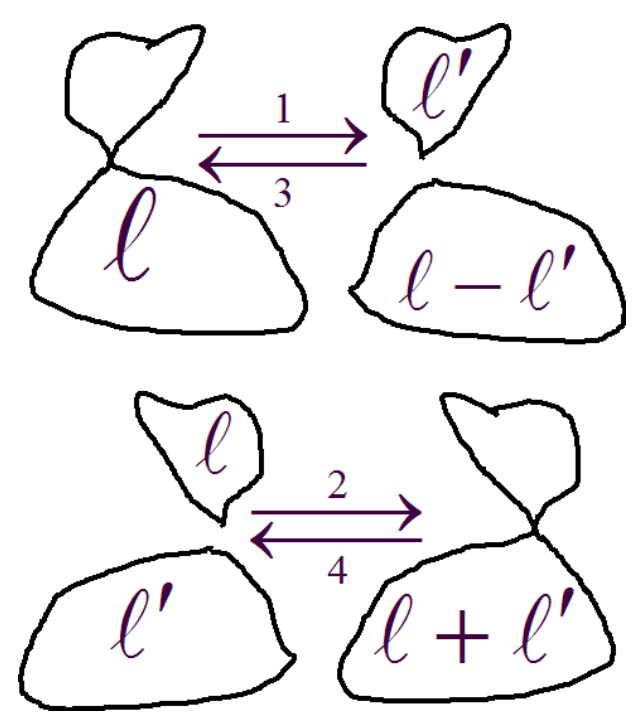

Figure 1: Strings joining and splitting. The number 1 refers to the first term on the right in Eq. (1), and so on.

The equilibrium condition $d n(\ell) / d t=0$ leads to the distribution $n(\ell)=$ $\frac{1}{\ell} e^{-\ell / L}$, where $L=\int_{0}^{\infty} d \ell \ell n(\ell)$. The strings are distributed such that, on average, there is one string at each length interval from $\ell=\ell_{i}$ to $\ell=e \ell_{i}$ 
(starting with $\ell_{i}=l_{s}$ and ending with $\ell_{i} \sim L / e$ ) up to $\ell=L$.

Let us define $N$ as the number of "string bits" of length $l_{s}$, so that $N=$ $L / l_{s}$. The string entropy is then essentially that of an $N$-step random walk, which is the logarithm of the number of different walks. If, for example, each step is \pm 1 , then $S=\ln 2^{N} \sim N$. The above distribution implies that most of the bits will be found on the longest strings, which will then make the dominant contribution to the entropy and energy, both of which scale with the total length of string.

\subsection{A bound state of highly excited strings: The col- lapsed polymer}

Our next objective is to describe the $\mathrm{BH}$ as a bound state of highly excited strings. Here, strong interactions lead to the formation of the bound state, whose volume is determined dynamically rather than by an external "box". As in the LT-SS description and as mentioned in the Introduction, flatspace coordinates, $t, r$, etc., will represent fiducial coordinates; labels for the strings in spacetime. In particular, $r$ is the radial fiducial coordinate with $r=0$ located at the center of the state. We will consider spherically symmetric configurations and let $r=R_{S}$ denote the finite spatial extent of the state. Although this need not be the Schwarzschild radius a priori, we will later show that it is parametrically equal to the Schwarzschild radius of the corresponding $\mathrm{BH}$.

When the strings are free and put in a large-enough volume, the radial size $R_{S}$ of the region is determined by the random-walk scale, which is $R_{S} \sim \sqrt{N}$ (in $l_{s}=1$ units) for a string of total length $N$. The joining and splitting 
of the closed strings lead, effectively, to an attractive interaction as they tend to make the strings occupy a smaller region in space. These attractive interactions therefore lead to a smaller value of $R_{S}$ [17, 38]. The free and interacting cases are depicted in Fig. 2. In general, in the absence of another scale besides that of $l_{s}$ (which is set to unity), we expect that $R_{S} \sim N^{\nu}$ for some $\nu$. The value of $\nu$ is determined by the strength of the string interactions. Since $N \sim R_{S}^{1 / \nu}$, the entropy will not, in general, be extensive in the volume. An area law, as in the case of BHs, implies that $\nu=1 /(d-1)$.

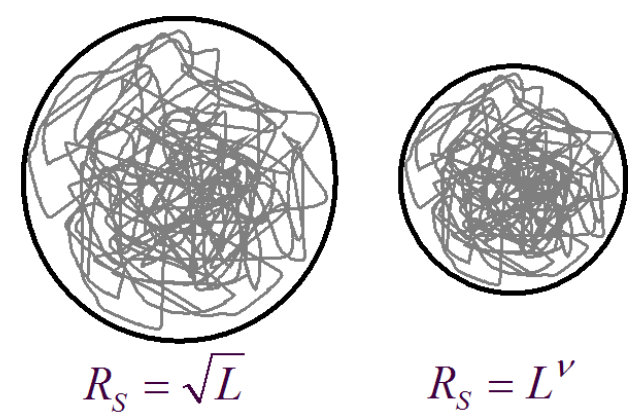

Figure 2: Strings in a bounded region. Free strings (left) occupy a larger region than interacting strings (right).

Such a scaling relation between the entropy and the spatial size of the state appears in the Flory-Huggins theory of polymers [40]. (See also the books by De Gennes [41] and Doi and Edwards [42].) This theory is reconsidered in [43] and reviewed, for example, in [44]. The transition temperature when the polymers become tensionless is known as the Flory temperature and $\nu$ is called the Flory exponent. The linear size $R_{S}$ is known as the gyration radius of the polymer. In the polymer literature, the case of attractive interactions is referred to as "negative excluded volume". In this case, the 
gyration radius of the polymer is smaller than $\sqrt{N}$ and the resulting state is called a "collapsed polymer".

The theory of collapsed polymers therefore provides an effective description of the bound state of highly excited strings, along with the appropriate adaptations which we now explain. The Flory effective free energy for the case of a collapsed polymer (see, e.g., [44]) is given by

$$
-\left(\frac{F}{V T}\right)_{\mathrm{CP}}=c-\frac{1}{2} v c^{2}+\mathcal{O}\left[c^{3}\right] .
$$

Here, $c$ is the entropy density (monomer concentration) and $v$ is the absolute value of the negative excluded volume. The relevance of the higher-order terms is controlled by the value of $v$. If the negative excluded volume is parametrically smaller than the volume $V \sim R_{S}^{d}$ occupied by the polymer, then the higher-order interaction terms are not important, just as in the SS-LT model.

The Flory theory then suggests an effective free energy for highly excited strings at a temperature close to but above the Hagedorn temperature, $T \gtrsim$ $T_{\text {Hag. }}$. In terms of the "bit concentration" or entropy density $c=N / V$ of the strings, this is

$$
-\left(\frac{F}{V T_{\text {Hag }}}\right)_{\text {strings }}=\epsilon c-\frac{1}{2} v c^{2}+\mathcal{O}\left[c^{3}\right]
$$

or, equivalently,

$$
-\left(\frac{F}{T_{\text {Hag }}}\right)_{\text {strings }}=\epsilon N-\frac{1}{2} \frac{v}{V} N^{2}+\mathcal{O}\left[\frac{N^{3}}{V^{2}}\right] .
$$

We will treat the Hagedorn temperature $T_{\text {Hag }}$ as equal to the string energy (ignoring an order-one numerical factor), so that $T_{\text {Hag }}=1$. 
We now would like to discuss the excluded volume $v$ and the additional (dimensionless) parameter $\epsilon$ for the specific case of highly excited strings. Let us begin with the latter parameter.

For this case of excited strings, we know that the leading-order expressions for the energy and entropy both scale with the number of string bits $N$; that is, $S \sim N$ and $E \sim N$ in string units. This is because the leading-order values should be comparable to those of the free-string case. Next consider that the free energy $F=-(S T-E)$ is, at leading order, expected to vanish at the Hagedorn temperature. It then follows that, for $T$ close to $T_{\text {Hag }}$, the difference $S T-E$ should be a number which is parametrically smaller than $N$. We have done, above, is to introduce a dimensionless parameter $\epsilon$ that parametrizes this small number. In fact, in light of Eq. (44), one can deduce that this parameter must of the form $\epsilon=\left(T-T_{H a g}\right) / T_{H a g} \ll 1$. This follows from $T \gtrsim T_{H a g}=1$ and then $\epsilon N \sim-F / T_{\text {Hag }}=(S T-E) / T_{\text {Hag }} \sim$ $\left(N T-N T_{H a g}\right) / T_{H a g}=N\left(T-T_{H a g}\right) / T_{H a g}$.

The second term on the right of Eq. (44) is meant to account for the effect of the interactions, which is parametrized by the excluded volume $v$ in the collapsed-polymer framework. In our case, the strength of the interactions is determined by $g^{2}$, and any one interaction is due to either the joining of a pair of strings or the splitting of a single string. These actions can take place whenever two bits of string occupy the same "point" in spacetime. Since the spacetime in question can be viewed as a lattice with a spacing of size $l_{s}$, the excluded volume can be identified as $v=g^{2} l_{s}^{d}=g^{2}$, and the density of coincident bits is then given by $g^{2} N^{2} / V$.

The effective free energy of the excited state of strings can now be ex- 
pressed as

$$
-\left(\frac{F}{V T_{\text {Hag }}}\right)_{\text {strings }}=\epsilon c-\frac{1}{2} g^{2} c^{2}+\mathcal{O}\left[c^{3}\right]
$$

or

$$
-\left(\frac{F}{T_{\text {Hag }}}\right)_{\text {strings }}=\epsilon N-\frac{1}{2} g^{2} \frac{N^{2}}{V}+\mathcal{O}\left[\frac{N^{3}}{V^{2}}\right],
$$

where, as discussed above, $\epsilon$ can be identified as the deviation of the temperature from the Hagedorn temperature, $\epsilon=\left(T-T_{\text {Hag }}\right) / T_{\text {Hag }}=T-1$. The value of $\epsilon$ need not be conjectured; its equilibrium value follows immediately from the minimization of the free energy, which gives (see below) $\epsilon=g^{2} N / V$. Then, insofar as the string state forms into a $\mathrm{BH}$, it is clear via the $\mathrm{BH}$ area law $N \sim R_{S}^{d-1} / g^{2}$ that, in string units, $\epsilon \sim 1 / R_{S} \sim T_{\text {Haw }}$.

The basic form $-F=S T-E$ tells that $\epsilon \sim T_{\text {Haw }}$ can indeed be viewed as an effective temperature for the bound state. Alternatively, $\epsilon$ can also be viewed as the relative increase in the excluded volume. That is, we can divide Eq. (4) through by $\epsilon$ and absorb $1 / \epsilon$ into $v$. From this point of view, $1 / \epsilon$ tells us how much that the excluded volume increases as the temperature increases from the Hagedorn value.

From the free energy in either Eq. (5) or (6) , one can obtain the equilibrium condition which follows from $\frac{\partial F}{\partial c}=0$. The solution, $c=\epsilon / g^{2}$, is valid only for finite coupling and is therefore "non-perturbative". In terms of $N$, the same equilibrium condition is

$$
\frac{N}{V}=\frac{\epsilon}{g^{2}}
$$

One can now evaluate the entropy density, energy density and pressure 
via the standard thermodynamic definitions. The results are as follows:

$$
\begin{aligned}
s & =-\left.\frac{1}{V} \frac{\partial F}{\partial \epsilon}\right|_{c=\epsilon / g^{2}}=\frac{\epsilon}{g^{2}} \\
\rho & =\left[\frac{F}{V}+\epsilon s\right]_{c=\epsilon / g^{2}}=\frac{1}{2} \frac{\epsilon^{2}}{g^{2}} \\
p & =-\left.\frac{\partial F}{\partial V}\right|_{c=\epsilon / g^{2}}=\frac{1}{2} \frac{\epsilon^{2}}{g^{2}} .
\end{aligned}
$$

Here, we have obtained the signature equation of state for a of high-temperature bath of closed strings, $p=\rho$.

In addition, one can calculate the effective tension and confirm that it vanishes at (and near) the Hagedorn or Flory temperature as expected,

$$
\sigma=\left.\frac{\partial F}{\partial L}\right|_{c=\epsilon / g^{2}}=\left.\frac{\partial F}{\partial N}\right|_{c=\epsilon / g^{2}}=0 .
$$

We can independently confirm the scaling relation $\epsilon \sim 1 / R_{S}$ from the fact that, at long distances, the string interactions are dominated by gravity [17, 38]. Hence, the total interaction energy of the bound state of strings is parametrically equal to its total gravitational energy,

$$
g^{2} \frac{N^{2}}{V} \sim G_{N} \frac{E^{2}}{R_{S}^{d-2}}
$$

where the left-hand side follows from the interaction term in the free energy (6) , or, equivalently, from an inspection of the LT Boltzmann Equation (11). The right-hand side is the Newtonian potential with $G_{N}=l_{P}^{d-1}=$ $g^{2} l_{s}^{d-1}=g^{2}$. The energy $E$ on the right side of Eq. (10) must be the boundstate energy $E=\rho V \sim \epsilon N$, and so

$$
\frac{R_{S}^{d-2}}{\epsilon^{2} V} \sim 1
$$


Then, since $V \sim R_{S}^{d}$, it follows that $\epsilon \sim 1 / R_{S}$, same as before.

The self-consistency of this framework necessitates the following set of hierarchies: $\epsilon \ll g^{2} \ll 1$ and $g^{2} N=V \epsilon \gg 1$ so that the $\mathrm{BH}$ is large in string units. These conditions ensure that the concentration is small, $c=\epsilon / g^{2} \ll 1$, which validates the expansion of the free energy and means that the higher-order terms can be neglected. Additionally, these conditions guarantee that the higher-order string interactions coming from $\alpha^{\prime}$ corrections, loop corrections or their combination are indeed suppressed. Nevertheless, they also ensure that $g^{2}$ is finite.

The relative strength of $(n+2)$-string interactions is proportional to $g^{2 n}$ and to a combinatoric enhancement factor $N^{n}$ and suppressed by a volume suppression factor $V^{-n}$. In string units, the multi-string interaction strength then goes as

$$
\lambda_{n+2} \sim\left(\frac{g^{2} N}{V}\right)^{n}=\epsilon^{n}=\left(\frac{l_{s}}{R_{S}}\right)^{n},
$$

where we have used Eq. (7). One can see that these are $\alpha^{\prime}$ corrections, being proportional to powers of $l_{s} / R_{S}$. Similarly, higher-order string-loop corrections and combinations thereof are suppressed, provided that $g^{2} \ll 1$.

Let us also recall that the size of a free string of a fixed length $N$ in string units is $\sqrt{N}=1 / g \epsilon^{(d-1) / 2}$, which follows from a random-walk scaling. Hence, the bound-state size of $1 / \epsilon$ is smaller than the free-string size by a factor of $g \epsilon^{(d-3) / 2} \ll 1$; meaning that the interactions are important, even though the higher-order interaction terms can be safely neglected.

The previous thermodynamic scaling relations agree with those of a $\mathrm{BH}$, provided that the bound-state energy is identified with the $\mathrm{BH}$ mass and the effective temperature with the Hawking temperature. For completeness, the 
dictionary goes as follows (in units in which $l_{s}=1$ and using only the dimensionless parameters $g^{2}$ and $\epsilon$ ): The volume of the interior is given by

$$
V=\frac{1}{\epsilon^{d}},
$$

the entropy by

$$
S_{B H}=N=V \frac{\epsilon}{g^{2}},
$$

the tension energy by

$$
E_{t e n}=N=V \frac{\epsilon}{g^{2}}
$$

and, correspondingly,

$$
T_{\text {Hag }}=1 .
$$

The total (bound-state) energy is

$$
M_{B H}=E_{\text {bound }}=V \frac{\epsilon^{2}}{g^{2}}
$$

and, correspondingly, the Hawking temperature is

$$
T_{\text {Haw }}=\frac{E_{\text {bound }}}{N}=\epsilon .
$$

In spite of the implicit presence of Planck's constant in its numerator, the Hawking temperature depends only on $\epsilon$ and not on $g^{2}$. This is so because Newton's constant cancels out of the ratio $M_{B H} / S_{B H}$.

\section{Emerging horizon and Hawking radiation}

As a gravitational concept, a horizon is a geometric realization of the fact that, classically, matter cannot escape from the interior of a BH. That is, a horizon can be defined, classically, as a closed surface through which matter 
is permitted to enter but not exit. Quantum mechanically, we expect two related effects to modify this picture.

For concreteness, we will consider an apparent BH horizon. According to its standard geometric and classical definition, an apparent horizon is a null spacetime surface where the effects of gravity are so strong that the escape velocity equals that of the speed of light. Consequently, incoming photons will end up orbiting this surface, whereas massive particles would inevitably fall in. Additionally, matter that is already inside this surface will never be able to escape, at least by classical means. However, from our viewpoint, the gravitational interactions in a curved spacetime have been replaced by inertial forces in a flat space, and so a dynamical description of the horizon is required.

Let us emphasize that the location of an apparent $\mathrm{BH}$ horizon is a physical observable since it is a measurable quantity (in principle). In [45], it is explained how an observer in a finite-sized laboratory can detect the horizon by measuring nearby tidal effects. For instance, an observer can measure the convergence of falling objects as a function of radial distance and use these measurements to determine where the curvature invariants coincide with their horizon values. This conclusion evades any violation of Einstein's equivalence principle because the principle is only relevant to ultra-local measurements.

How would a bound state of highly excited strings in a finite region of space - a "ball of string" - form a dynamical horizon? Classically, the strings cannot split, as the spliting of strings is a quantum effect in string theory. If the strings cannot split, bits of string cannot break off and escape 
from the ball of string. Our new definition of the horizon is then the smallest spherical shell that entirely encloses the ball of string. The size of this shell can, classically, be expected to be set by the random-walk scale of a free string. Then, just like the standard geometric definition of a horizon, this dynamically formed shell effectively describes a one-way barrier to the external world.

Once quantum mechanics is taken into account, the standard definition of a $\mathrm{BH}$ horizon becomes less sharp. The reason for this is two-fold: First, the position of the horizon is expected to fluctuate, as does, generically, any other physical observable. Second, the process of Hawking radiation.

Regarding fluctuations, we have previously shown [28, 29, 30] that the quantum variance of $R_{S}$ scales as

$$
\frac{\left(\Delta R_{S}\right)^{2}}{\left\langle R_{S}\right\rangle^{2}} \simeq \frac{1}{S_{B H}}
$$

This is a general result that is applicable in any dimension and follows from simple quantum-mechanical considerations: Whatever quantum wavefunction correctly describes a $\mathrm{BH}$, it must yield certain expectation values in the classical limit according to the Bohr correspondence principle. Clearly, the expectation value of the "entropy operator" $\widehat{S}$ (See [28, 29, 30] for the precise definition) must correspond to the Bekenstein-Hawking entropy $S_{B H}$. As for the variance of the entropy operator, standard thermodynamics dictates that this is given by the magnitude of the heat capacity, which is also of the order of $S_{B H}$. It then follows that

$$
\frac{(\Delta \widehat{S})^{2}}{\langle\widehat{S}\rangle^{2}} \simeq \frac{1}{S_{B H}},
$$

from which Eq. (19) can be obtained by way of the chain rule. 
That $1 / S_{B H}$ acts as a dimensionless $\hbar$ is also apparent in the context of the AdS/CFT duality: The quantity that controls the strength of quantum fluctuations on the field-theory side is $1 / \mathcal{N}^{2}$, which on the gravity side corresponds to $1 / S_{B H}$ (here, $\mathcal{N}$ is the number of colors).

The second quantum effect to consider is the emission of Hawking radiation. Quantum mechanically, matter can escape from the $\mathrm{BH}$ due to the Hawking process. The rate and average energy of the escaped particles is determined by the size of the $\mathrm{BH}, R_{S}$.

The purpose of this section is to verify that the general picture that we just presented can be reproduced from the specific microscopic model of the collapsed polymer. This means establishing the emergence of a classical horizon that fluctuates quantum mechanically at the correct strength as in Eq. (19). Also, that strings escape from the bound state via a quantummechanical effect whose rate and energy of emission agree with those of the Hawking process. Since the microscopic model does not contain gravity explicitly, this constitutes a highly non-trivial check on the viability of our proposal for the interior.

\subsection{Emerging Horizon}

Let us recall the Boltzmann-like equation for interacting, closed strings, Eq. (11). The string interaction strength is proportional to $g^{2}$ [23, 24]. So that, in the classical approximation, the coupling $g^{2}$ vanishes and pieces of the polymer cannot detach from its main body. This is the simplest expression of a horizon, as the escape velocity of a piece must be infinitely large as long as it is still connected to the polymer. The only question in this case is 
how far away can a piece get? Perhaps it can still escape to infinity for all practical purposes.

A related question is to what extent is the size of the polymer well defined? This leads to a more precise criterion for the existence of a horizon: The extent to which pieces of the polymer can protrude away from the main body in relation to its diameter $2 R_{S}$. What we then need to estimate is the strength of the fluctuations in the position of the horizon and check that these are sufficiently weak.

To this end, let us recall Eq. (5) for the effective free energy $F$ in terms of the polymer concentration $c$, where $c=N / V$ at equilibrium. Rearranging slightly (and using $T_{H a g=}=1 / l_{s}=1$ ), we have

$$
\frac{F}{\epsilon}=-c V+\frac{1}{2} V \frac{g^{2}}{\epsilon} c^{2}+\mathcal{O}\left[c^{3}\right]
$$

Since $c$ is conjugate to the volume $V$ in Eq. (21), one can apply standard statistical mechanics and determine the variance of the volume fluctuations by twice varying the free energy with respect to the polymer concentration. That is,

$$
(\Delta V)^{2}=\frac{\partial^{2}(F / \epsilon)}{\partial c^{2}}=\frac{V g^{2}}{\epsilon} .
$$

Using the equilibrium relation in Eq. (7), $\epsilon=g^{2} N / V$, and dividing through by $V^{2}$, we now obtain

$$
\frac{(\Delta V)^{2}}{V^{2}}=\frac{1}{N}=\frac{1}{S_{B H}} .
$$

Then, by way of the chain rule, the boundary or horizon fluctuations scale according to

$$
\frac{\left(\Delta R_{S}\right)^{2}}{R_{S}^{2}} \simeq \frac{1}{N}
$$


just as expected for a $\mathrm{BH}$ with an entropy of $N$. Clearly, this is the smallest possible variance, being that it is proportional to $1 / S_{B H}$, the dimensionless $\hbar$ for this framework. Thus, the position of the horizon is as well defined as it can be.

\subsection{Hawking radiation}

The viability of quantum escape can be determined in two steps: By, first, calculating the rate at which small strings escape (see Fig. 3) and, second, determining the suppression factor for the rate of escape of longer strings with respect to the small strings. Throughout this subsection, we use units in which $l_{s}=1$.

To address the first step, let us start by recalling that the equilibrium solution of Eq. (11) for the string-number function is [24]

$$
n(\ell)=\ell^{-1} e^{-\frac{\ell}{L}}
$$

where $L$ is the expectation value of the total length of string (i.e., $L=N$ in string units). It follows that, on average, the number of the shortest strings loops of unit length - is approximately $n(1) \simeq 1$. We can therefore assume that there is only a single short string at any given time.

The probability that a short string can survive its journey from an arbitrary location in the polymer to the outer surface depends mainly on its mean-free-path length $l_{m f p}$. Once a small string does safely reach the surface, it has an order-unity probability of escaping if its velocity contains an outward component. On the other hand, if the short string instead interacts, it will join onto a longer string, and whether or not this longer loop can make 


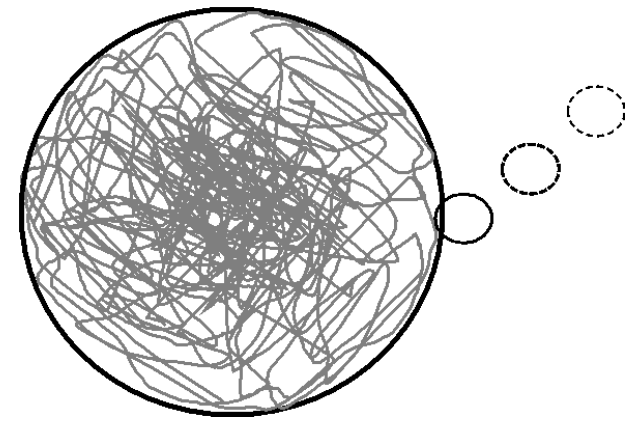

Figure 3: A dynamical Hawking process: Small loops can tear off and escape from the bulk of the polymer.

it to the surface is a case which will be discussed separately.

It is easier to begin with the mean-free-path time, $t_{m f p}=l_{m f p} / v_{C o M}$ with $v_{C o M}$ denoting the speed of the string's center of mass. The mean-freepath time for a string of length $\ell$ can be determined by way of the following relation:

$$
\frac{d E}{d t}=\frac{g^{2} N}{V} E(\ell)
$$

where the left-hand side is the power absorbed by the string because of interactions and $E(\ell) \propto \ell$ is its energy. An expression of this basic form follows from $g^{2} N / V$ measuring the overall strength of the interactions and from the interaction rate of any given string being proportional to its length [23, 24].

We can estimate $t_{m f p}$ for a short string by defining it as the time when the absorbed energy becomes of the same order as its original energy $E(1)$. This will happen when

$$
t_{m f p} \simeq \frac{V}{N g^{2}}=\frac{1}{\epsilon}
$$


where we have recalled the equilibrium condition $V / N g^{2}=1 / \epsilon$ in Eq. (7). Since a string with a length of order one can be regarded as relativistic, it follows that

$$
l_{m f p}=t_{m f p} \simeq \frac{1}{\epsilon}
$$

for the shortest strings.

As $1 / \epsilon=R_{S}$, the result in Eq. (28) means that a short string has an order-one probability of making to the surface. And, since there is (on average) only one such string at any given time, the rate of escape of the short strings is just the inverse of the mean-free-path time, $1 / t_{m f p}=\epsilon$. This is exactly the rate at which $\mathrm{BHs}$ emit their radiation because $T_{\text {Haw }}=\epsilon$ determines the emission rate of the Hawking particles. And so the rate of escape of strings whose length is of order one is

$$
\frac{1}{t_{m f p}} \simeq T_{H a w}
$$

We can therefore conclude that the emission of Hawking radiation is a consequence of the smallest possible loops of string (on the order of one string length) escaping from the collapsed polymer. This fits well with energetic considerations, as a single bit of string will carry a net energy of about

$$
E_{\text {emitted }}=\frac{M_{B H}}{N}=\epsilon=T_{\text {Haw }}
$$

away from the bound state. Therefore, if only short strings contribute, the rate of energy emission is

$$
\frac{d E_{\text {emitted }}}{d t} \simeq \epsilon^{2}=T_{\text {Haw }}^{2}
$$

This energy budgeting may seem contradictory to a just-escaped string having a tension of order one. However, like any particle in a bound state or 
a potential well, it expends some energy in climbing out of the well. Here, we know the average of how much energy is typically retained by such a string; it is simply $E_{\text {bound }} / N=\epsilon$. Also note that there is effectively no Tolman redshift in this calculation because of our choice to describe gravity as an inertial force in a flat spacetime.

We do not mean, of course, that an escaped string will directly transform into a physical Hawking particle. Once in the exterior, the string is quickly subsumed into the external string condensate which describes the classical gravitational field. The condensate will then rearrange to accommodate this new excitation; an action which is manifested in the physical spacetime as the emission of a Hawking particle. An external observer would naturally attribute this process to one of gravitationally induced pair production [46]. Meanwhile, the internal polymer rearranges to accommodate the loss of the string, finally settling into a new equilibrium state with a slightly lower energy. The external observer would attribute this reduction in energy to the absorption of the negative-energy pair partner of the emitted particle.

What still needs to be checked is the fate of strings that are parametrically longer than the fundamental string length. If these strings escape at a significant rate, then energy will be leaking faster from the polymer than it would be for a BH. Fortunately, there is a simple statistical argument which ensures that the escape rate of long strings is exponentially suppressed. 2

We can use entropic considerations to estimate the probability that a long string of length $\ell \gg 1$ detaches from the body of the polymer. A standard argument in statistical mechanics maintains that, near equilibrium, the rel-

\footnotetext{
${ }^{2}$ We thank Masanori Hanada for suggesting this argument.
} 
ative probability of a string of total length $L$ splitting into two disconnected strings - one of length $L-\ell$ and the other of length $\ell$ - decreases exponentially with the entropy of the detached string, which is equal to its length $\ell$ in string units. It follows that the probability of detachment goes as

$$
\frac{P(L-\ell, \ell)}{P(L)} \sim e^{-\ell}
$$

It can be concluded that the total emitted energy from all long strings of any length is suppressed by an exponentially small factor with respect to that of the shortest strings. 3 Therefore, the long strings make a negligible contribution.

\section{Chaos, fast scrambling and viscosity}

The main objective of this section is to understand how (and if) the collapsed polymer is able to reproduce the maximally fast scrambling rate [31, 32, 33 . and the minimally allowed viscosity-to-entropy ratio [35, 36, 37] of a $\mathrm{BH}$. From the BH perspective, these properties pertain to just the horizon, but we will be able to make a novel prediction about the latter one in the interior. The key idea in this section is the use of standard statistical mechanics to relate the polymer free energy to the various quantities of interest.

\footnotetext{
${ }^{3}$ There will also be an additional power-law suppression due to longer strings being fewer in number ( $c f$, Eq. (25)), having a shorter mean-free-path length and a nonrelativistic velocity.
} 


\subsection{Chaos and fast scrambling}

In the context of BHs, the scrambling time is meant to be the typical time scale for a disturbance that is induced by in-falling matter to disperse over the entire horizon surface. Underlying this definition is the premise that an external observer can never see matter or energy fall into the $\mathrm{BH}$ - so that, as far as she is concerned, any such disturbance is forever trapped on the horizon. 4 But the internal perspective, which must be adopted in our framework, is necessarily somewhat different and more similar to the way that the scrambling time is defined in systems which can be accessed by an observer.

One might wonder about the (prospective) physical mechanism that underlies rapid scrambling in a bound state of strings. Any perturbation of the bound state away from its equilibrium configuration must propagate along a trajectory that follows the path of one of the strings. Nevertheless, spacetime points nearby one another can live on different strings (or on distinct portions of the same long string) and, therefore, nearby modes will tend to propagate along completely different trajectories following completely different directions. In this way, a perturbation of one small region of the string state can quickly spread out to a much larger region because of the divergent paths of the resulting waves. This effect is depicted in Fig 4 .

Despite being very far apart on a single long string, string bits can be very close in spacetime and therefore can interact. In other words, the string interactions are effectively non-local from the string point of view and a

\footnotetext{
${ }^{4}$ It is more conventional to frame the scrambling time in terms of the "stretched horizon", which is taken to be a few Planck lengths outward from the actual horizon.
} 


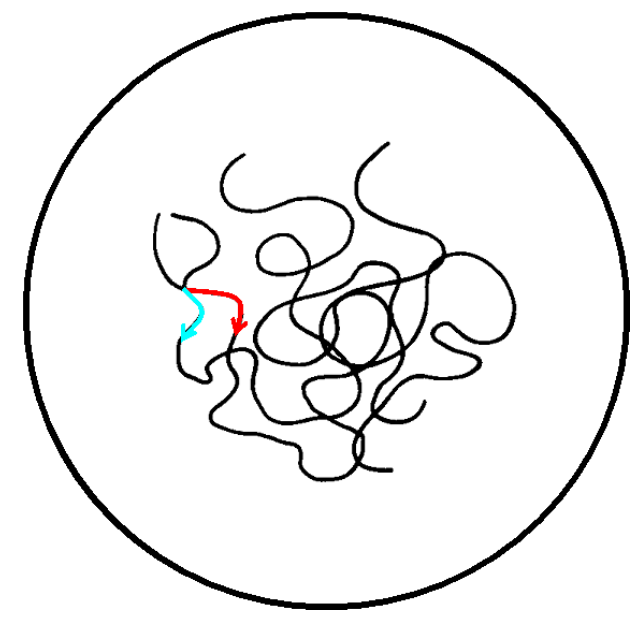

Figure 4: A perturbation of bits of string in the same spacetime region but on different strings (or on well-separated parts of the same string) will diverge by propagating on the one-dimensional strings.

disturbance can be expected to delocalize on the string in a short time scale. But is this "delocalization time" the same as the minimally short time scale that is saturated by a perturbed $\mathrm{BH}$ ?

In general, the scrambling time can be associated with the time scale for the onset of fully chaotic behavior in a perturbed dynamical system, and it is determined (in part) by the largest positive Lyapunov exponent $\lambda_{L}$. (See, e.g., [34, 47, 48] for recent discussions in the context of BHs.) The exponent $\lambda_{L}$ appears in an exponentially growing factor that is associated with the degree of delocalization of an initial disturbance. In this case, the "delocalization" is meant to be taking place in phase space and can be quantified in terms of the increasing "distance" between once-neighboring trajectories. The maximal amount of delocalization is set by the size of the accessible 
region of phase space.

In a quantum theory, one of the standard methods of diagnosing for chaos and then identifying the Lyapunov exponent is to inspect an out-of-timeorder four-point function for a suitable choice of unitary operators. With this in mind, we will apply an argument from [47] that reveals how the scrambling time can be deduced for any chaos-prone quantum theory. The starting point is the following observation about chaotic motion in phase space: If $q\left(t ; q_{0}, p_{0}\right)$ and $p\left(t ; q_{0}, p_{0}\right)$ are a canonically conjugate pair describing trajectories in phase space $\left(q_{0}\right.$ and $p_{0}$ are their initial values), it can be expected that

$$
\frac{\partial q(t)}{\partial q_{0}} \sim e^{\lambda_{L} t}
$$

Now suppose that $U(t)=e^{i \alpha q(t)}$ and $V(t)=e^{i \beta p(t)}$, where $\alpha$ and $\beta$ are dimensional constants. Insofar as $\alpha, \beta$, and $t$ are suitably small, then Eq. (33) plus some basic quantum mechanics leads to

$$
\left\langle U^{\dagger}(t) V^{\dagger}(0) U(t) V(0)\right\rangle \sim \exp \left[-i \alpha \beta \hbar \exp \left(\lambda_{L} t\right)\right]
$$

where we have temporarily restored Planck's constant to emphasize that the product $\alpha \beta$ has units of inverse action. A large phase in an out-of-time-order four-point function can be viewed as a signal of chaotic behavior. Here, the phase becomes order unity when

$$
t \sim-\frac{1}{\lambda_{L}} \ln (\alpha \beta \hbar)
$$

The scrambling time $t_{s c r}$ can be identified as the time for which a disturbance is delocalized throughout phase space. Therefore, $t_{s c r}$ can be estimated by identifying the measure of action $(\alpha \beta)^{-1}$ with the size of the phase space 
in question. Our model has a phase space of size $N \hbar$ since the number of interacting degrees of freedom is $N$ and they all interact with each other in a democratic way. Consequently,

$$
t_{s c r} \sim \frac{1}{\lambda_{L}} \ln N
$$

What is left is to determine the Lyapunov exponent $\lambda_{L}$. To do this, we will adopt a commonly used method from statistical mechanics which relates the value of the free energy to the Lyapunov exponent. In the standard treatment, one calculates the transfer matrix $T$ (see, e.g., Section 10 of [49], as well as [50]) and determines its largest eigenvalue $\lambda_{+}$. The Lyapunov exponent $\lambda_{L}$ can then be identified with the logarithm of this largest eigenvalue, $\lambda_{L}=\ln \lambda_{+}$(see, e.g., [51]). The argument uses two different representations of the partition function $Z$,

$$
Z=\operatorname{Tr} T_{1}^{N}
$$

and

$$
Z=\operatorname{Tr} e^{-\beta F}
$$

where $\beta$ is the inverse temperature and $T_{1}$ is the transfer matrix for a single degree of freedom. The transfer matrix acts in Euclidean time over an interval of length $\beta$.

Let us denote the number of degrees of freedom (more generally, the entropy) by $N$. Then, for large $N$,

$$
Z \sim \lambda_{+}^{N}=e^{\beta \lambda_{L} N}
$$

where $\lambda_{+}=e^{\beta \lambda_{L}}$. Comparing Eqs. (38) and (39), we see that the Lyapunov exponent can then be expressed in terms of the free energy per degree of 
freedom $F / N$,

$$
\beta \lambda_{L}=-\frac{\beta F}{N} .
$$

Let us recall that the free energy of the polymer can be expressed at equilibrium as

$$
-\frac{F}{T_{\text {Hag }} N}=\epsilon-\frac{1}{2} \frac{g^{2} N}{V}=\frac{1}{2} \epsilon
$$

and that $\epsilon=1 / R_{S}$. It can therefore be concluded that

$$
\lambda_{L}=\frac{1}{2} \epsilon=\frac{1}{2 R_{S}} .
$$

Using the standard definition of the Hawking temperature $T_{\text {Haw }}=$ $1 /\left(4 \pi R_{S}\right)$ (which also follows from the free energy in Eq. (41)), we have

$$
\lambda_{L}=2 \pi T_{\text {Haw }} .
$$

And so the Lyapunov exponent can be identified with the inverse of the Hawking thermal wavelength.

We would like to emphasize that our derivation of the Lyapunov exponent follows from its standard definitions for dynamical systems and does not rely on the ratio of redshifts between different observers. Indeed, gravity never enters into our calculation.

A positive value for $\ln \lambda_{+}$means that the maximal eigenvalue of the transfer matrix is larger than unity and serves as a standard indicator of chaotic behavior. Further, for a strongly interacting system at a finite temperature, the Lyapunov exponent should be close to but bounded from above by the inverse of the thermal wavelength [34]. For our model, this bound appears to be saturated, just as expected for a $\mathrm{BH}$. 
One can now be conclude, using Eqs. (43) and (36), that the scrambling time for our model is indeed the anticipated expression [31, 32, 33,

$$
t_{s c r}=\frac{1}{2 \pi T_{H}} \ln S_{B H} .
$$

An alternative method for estimating the Lyapunov exponent goes as follows: The inverse of the Lyapunov exponent is associated with the time scale for a system to equilibrate or thermalize. We can estimate this time scale by considering a perturbation away from equilibrium of the distribution of strings for some length of string $\ell_{0}$. Let us then recall the Boltzmann-like equation (11) and consider $n\left(\ell_{0}\right) \rightarrow n\left(\ell_{0}\right)+\delta n\left(\ell_{0}\right)$. It can be shown that such a perturbation dissipates in time according to [24]

$$
\frac{\delta n\left(\ell_{0}, t\right)}{\delta n\left(\ell_{0}, 0\right)}=e^{-a \ell_{0} \frac{g^{2}}{V}\left(N+\frac{\ell_{0}}{2}\right) t} \simeq e^{-a \ell_{0} \frac{g^{2} N}{V} t},
$$

where $a$ is some order-unity numerical factor in string units.

The longest equilibration time for the string state should be determined by adding the shortest possible string, which means setting $\ell_{0}=1$ in the exponent of Eq. (45). It follows that

$$
\lambda_{L}=a \frac{g^{2} N}{V}=a \epsilon=\frac{a}{R_{S}} .
$$

Causality requires that $a \leq 1$, and so

$$
\lambda_{L} \lesssim \frac{1}{R_{S}} .
$$

The previous discussion indicates that $a=1 / 2$.

\subsection{Viscosity to entropy ratio}

The discussion in the previous subsection regarding dissipation will enable us

to estimate the shear viscosity $\eta$ for the stringy fluid within the interior. The 
viscosity can, in general, be identified with the ratio of the energy density $\rho=\epsilon^{2} / g^{2}$ and the time scale for dissipation $\lambda_{L}$. So that, in our case,

$$
\eta \simeq \frac{\rho}{\lambda_{L}}=\frac{2}{\epsilon} \frac{\epsilon^{2}}{g^{2}},
$$

where the second equality follows from Eqs. (17) and (43). Using Eq. (77), we then have

$$
\eta \simeq \frac{2 \epsilon}{g^{2}}=2 \frac{N}{V}=2 s .
$$

Our conclusion is that the shear viscosity is the same order as the entropy density, $\eta / s \simeq 1$. This is true not only at the horizon, as expected [35, 36, 37, but throughout the entirety of the interior region!

It is amusing to consider the following method for fixing $\eta$ with precision. For certain physical situations, the shear viscosity of a fluid can be determined by an expression of what is basically the form (see, e.g., [52])

$$
\eta=2 \frac{T}{\gamma^{2}} \lambda_{L} s,
$$

where $\gamma$ is the shear rate which has dimensions of velocity divided by length.

The maximum possible shear rate is when the velocity is that of light and the length in question is that of the system. In our case, the maximal shear rate would then be given by the inverse radius

$$
\gamma_{\max }=1 / R_{S} .
$$

It could be expected, based on the previous discussion, that the stringy fluid does indeed saturate the maximal value of the shear rate. Then, substituting for $\lambda_{L}$ from Eq. (43) and setting $T=T_{\text {Haw }}$, we find that

$$
\eta=\frac{1}{4 \pi} s,
$$


which precisely saturates the celebrated bound of Kovtun, Son and Starinets [37]. It follows that the maximal value of $\lambda_{L}$ corresponds to the minimal value of $\eta$, just as one would anticipate.

This uniform saturation of what is believed to be an absolute lower bound on the viscosity-to-entropy ratio is a novel prediction of our model. It also further supports the notion that the interior of the $\mathrm{BH}$ is a place where semiclassical physics breaks down, as the saturation of this lower bound is supposed to be a signature of strongly coupled field theories [34.

\section{Conclusion}

We have revisited our recent proposal that the $\mathrm{BH}$ interior is a bound state of long, highly excited, closed strings or, equivalently, a string-theory version of a collapsed polymer. After reviewing some of the model's salient features, we introduced several new consistency checks for this paradigm. We showed that the position of the outer surface fluctuates quantum mechanically with the expected relative variance of $1 / S_{B H}$. We also demonstrated that the process of Hawking radiation — with its anticipated rate of emission and energy per emitted particle — can be attributed to the quantum effect of small strings escaping from the main body of the polymer . In other words, the $\mathrm{BH}$ horizon emerges as a natural feature in the polymer framework.

In addition, we have applied standard methods from statistical mechanics and chaos theory and found values for the Lyapunov exponent, scrambling time and viscosity-to-entropy ratio that saturate their respective bounds and, therefore, agree with the standard expected results for BHs. A novel feature 
of our model is that the viscosity-to-entropy ratio saturates its minimally allowed value throughout the interior and not just at the horizon. One of our next objectives [53] is to find a novel prediction that could potentially be verified by the (anticipated) data coming from gravitational-wave detectors [54.

\section{Acknowledgments}

We would like to thank Yshai Avishai, Doron Cohen, Guy Gur-Ari, Masanori Hanada and Sunny Itzhaki for valuable discussions. The research of RB is supported by the Israel Science Foundation grant no. 1294/16. The research of AJMM received support from an NRF Incentive Funding Grant 85353, an NRF Competitive Programme Grant 93595 and Rhodes Research Discretionary Grants. AJMM thanks Ben Gurion University for their hospitality during his visit.

\section{References}

[1] S. D. Mathur, "What Exactly is the Information Paradox?," Lect. Notes Phys. 769, 3 (2009) [arXiv:0803.2030 [hep-th]].

[2] S. D. Mathur, "The Information paradox: A Pedagogical introduction," Class. Quant. Grav. 26, 224001 (2009) [arXiv:0909.1038 [hep-th]].

[3] S. D. Mathur, "What the information paradox is not," arXiv:1108.0302 [hep-th]. 
[4] S. D. Mathur, "What does strong subadditivity tell us about black holes?," Nucl. Phys. Proc. Suppl. 251-252, 16 (2014) [arXiv:1309.6583 [hep-th]].

[5] K. Skenderis and M. Taylor, "The fuzzball proposal for black holes," Phys. Rept. 467, 117 (2008) [arXiv:0804.0552 [hep-th]].

[6] A. Almheiri, D. Marolf, J. Polchinski and J. Sully, "Black Holes: Complementarity or Firewalls?," JHEP 1302, 062 (2013) [arXiv:1207.3123 [hep-th]].

[7] N. Itzhaki, "Is the black hole complementarity principle really necessary?," arXiv:hep-th/9607028.

[8] S. L. Braunstein, S. Pirandola and K. Zyczkowski, "Entangled black holes as ciphers of hidden information," Physical Review Letters 110, 101301 (2013) [arXiv:0907.1190 [quant-ph]].

[9] D. Marolf and J. Polchinski, "Gauge/Gravity Duality and the Black Hole Interior," Phys. Rev. Lett. 111, 171301 (2013) [arXiv:1307.4706 [hep-th]].

[10] R. Brustein and A. J. M. Medved, "Black holes as collapsed polymers," arXiv:1602.07706 [hep-th].

[11] L. Alberte, R. Brustein, A. Khmelnitsky and A. J. M. Medved, "Density matrix of black hole radiation," JHEP 1508, 015 (2015) [arXiv:1502.02687 [hep-th]]. 
[12] S. Fubini and G. Veneziano, "Level structure of dual resonance models," Nuovo Cimento 64A, 1640 (1969).

[13] D. Mitchell and N. Turok, "Statistical mechanics of cosmic strings," Phys. Rev. Lett. 58, 1577 (1987); "Statistical properties of cosmic strings," Nucl. Phys. B 294, 1138 (1987).

[14] J. J. Atick and E. Witten, "The Hagedorn transition and the number of degrees of freedom in string theory," Nucl. Phys. B 310, 291 (1988).

[15] N. Deo, S. Jain and C.-I. Tan "String distributions above Hagedorn energy density," Phys. Rev. D 40, 2626 (1989).

[16] R. Brandenberger and C. Vafa, "Superstrings in the early universe," Nucl. Phys. B 316, 391 (1989).

[17] G. T. Horowitz and J. Polchinski, "Selfgravitating fundamental strings," Phys. Rev. D 57, 2557 (1998) [hep-th/9707170].

[18] J. D. Bekenstein, "Black holes and entropy," Phys. Rev. D 7, 2333 (1973).

[19] S. W. Hawking, "Black hole explosions", Nature 248, 30 (1974); "Particle creation by black holes", Comm. Math. Phys. 43, 199 (1975).

[20] R. Brustein and A. J. M. Medved, "Quantum state of the black hole interior," JHEP 1508, 082 (2015) [arXiv:1505.07131 [hep-th]].

[21] R. Brustein and G. Veneziano, "A Causal entropy bound," Phys. Rev. Lett. 84, 5695 (2000) [hep-th/9912055]. 
[22] R. Brustein and M. Schmidt-Sommerfeld, "Universe Explosions," JHEP 1307, 047 (2013) [arXiv:1209.5222 [hep-th]].

[23] P. Salomonson and B.-S. Skagerstam, "On superdense superstring gases: A heretic string model approach," Nucl. Phys. B 268, 349 (1986).

[24] D. A. Lowe and L. Thorlacius, "Hot string soup," Phys. Rev. D 51, 665 (1995) [hep-th/9408134].

[25] L. Susskind, "Some speculations about black hole entropy in string theory," In *Teitelboim, C. (ed.): The black hole* 118-131 [hepth/9309145].

[26] G. T. Horowitz and J. Polchinski, "A Correspondence principle for black holes and strings," Phys. Rev. D 55, 6189 (1997) doi:10.1103/PhysRevD.55.6189 [hep-th/9612146].

[27] T. Damour and G. Veneziano, "Selfgravitating fundamental strings and black holes," Nucl. Phys. B 568, 93 (2000) doi:10.1016/S05503213(99)00596-9 [hep-th/9907030].

[28] R. Brustein and M. Hadad, "Wave function of the quantum black hole," Phys. Lett. B 718, 653 (2012) [arXiv:1202.5273 [hep-th]].

[29] R. Brustein, "Origin of the blackhole information paradox," Fortsch. Phys. 62, 255 (2014) [arXiv:1209.2686 [hep-th]].

[30] R. Brustein and A. J. M. Medved, "Semiclassical black holes expose forbidden charges and censor divergent densities," JHEP 1309, 108 (2013) [arXiv:1302.6086 [hep-th]]. 
[31] P. Hayden and J. Preskill, "Black holes as mirrors: Quantum information in random subsystems," JHEP 0709, 120 (2007) [arXiv:0708.4025 [hep-th]].

[32] Y. Sekino and L. Susskind, "Fast Scramblers," JHEP 0810, 065 (2008) [arXiv:0808.2096 [hep-th]].

[33] L. Susskind, "Addendum to Fast Scramblers," arXiv:1101.6048 [hep-th].

[34] J. Maldacena, S. H. Shenker and D. Stanford, "A bound on chaos," arXiv:1503.01409 [hep-th].

[35] K. S. Thorne, R. H. Price, D. A. Macdonald, Black Hole: The Membrane Paradigm, New Haven (Yale University Press, London, 1986).

[36] G. Policastro, D. T. Son and A. O. Starinets, "The Shear viscosity of strongly coupled N=4 supersymmetric Yang-Mills plasma," Phys. Rev. Lett. 87, 081601 (2001) [hep-th/0104066].

[37] P. Kovtun, D. T. Son and A. O. Starinets, "Viscosity in strongly interacting quantum field theories from black hole physics," Phys. Rev. Lett. 94, 111601 (2005) [hep-th/0405231].

[38] T. Damour and G. Veneziano, "Selfgravitating fundamental strings and black holes," Nucl. Phys. B 568, 93 (2000) [hep-th/9907030].

[39] D. Chialva, "Self-interacting fundamental strings and black holes," Nucl. Phys. B 819, 256 (2009) [arXiv:0903.3977 [hep-th]]. 
[40] P. J. Flory, Principles of Polymer Chemistry (Cornell University, Ithaca, New York, 1953).

[41] P. G. De Gennes, Scaling Concepts in Polymer Physics (Cornell University, Ithaca, New York, 1979).

[42] M. Doi and S. F. Edwards, The Theory of Polymer Dynamics (Oxford University Press, Oxford, U.K., 1986).

[43] P. G. De Gennes, "Collapse of a polymer chain in poor solvents," Journal de Physique Lettres 36, L55 (1975).

[44] C. Williams, F. Brochard and H. L. Frisch, "Polymer Collapse," Ann. Rev. Phys. Chem. 32, 433 (1981)

[45] M. Visser, "Physical observability of horizons," Phys. Rev. D 90, no. 12, 127502 (2014) [arXiv:1407.7295 [gr-qc]].

[46] S. W. Hawking, "Breakdown of Predictability in Gravitational Collapse," Phys. Rev. D 14, 2460 (1976).

[47] B. Swingle, G. Bentsen, M. Schleier-Smith and P. Hayden, "Measuring the scrambling of quantum information," arXiv:1602.06271 [quant-ph].

[48] B. Michel, J. Polchinski, V. Rosenhaus and S. J. Suh, "Fourpoint function in the IOP matrix model," JHEP 1605, 048 (2016) [arXiv:1602.06422 [hep-th]].

[49] K. G. Wilson and J. B. Kogut, "The Renormalization group and the epsilon expansion," Phys. Rept. 12, 75 (1974). doi:10.1016/03701573(74)90023-4 
[50] M. Creutz, "Gauge Fixing, the Transfer Matrix, and Confinement on a Lattice," Phys. Rev. D 15, 1128 (1977). doi:10.1103/PhysRevD.15.1128

[51] A. MacKinnon and B. Kramer, "One-Parameter Scaling of Localization Length and Conductance in Disordered Systems," Phys. Rev. Lett. bf 47, 1546 (1981);

P. S. Davids, "Lyapunov exponent and transfer-matrix spectrum of the random binary alloy," Phys. Rev. B 52, 4146 (1995).

[52] D. Evans, E. G. D. Cohen and G. P. Morriss, "Viscosity of a simple fluid from its maximal Lyapunov exponents," Phys. Rev. A 42, 5990 (1990).

[53] R. Brustein and A. J. M. Medved, in preparation.

[54] B. P. Abbott et al. [LIGO Scientific and Virgo Collaborations], "Observation of Gravitational Waves from a Binary Black Hole Merger, Phys. Rev. Lett. 116, no. 6, 061102 (2016) [arXiv:1602.03837 [gr-qc]]. 